\title{
The utility of automated volume analysis of renal stones before and after shockwave lithotripsy treatment
}

\author{
Helen Wei Cui ${ }^{1} \mathbb{D} \cdot$ Tze Khiang Tan $^{2}$ · Frederikke Eichner Christiansen ${ }^{3} \cdot$ Palle Jörn Sloth Osther $^{3}$. \\ Benjamin William Turney ${ }^{1}$
}

Received: 28 March 2020 / Accepted: 31 August 2020 / Published online: 14 September 2020

(c) The Author(s) 2020

\begin{abstract}
This study aimed to evaluate the additional utility of an automated method of estimating volume for stones being treated with shockwave lithotripsy (SWL) using computed tomography (CT) images compared to manual measurement. Utility was assessed as the ability to accurately measure stone burden before and after SWL treatment, and whether stone volume is a better predictor of SWL outcome than stone diameter. 72 patients treated with SWL for a renal stone with available CT scans before and after treatment were included. Stone axes measurement and volume estimation using ellipsoid equations were compared to volume estimation using software using CT textural analysis (CTTA) of stone images. There was strong correlation $(r>0.8)$ between manual and CTTA estimated stone volume. CTTA measured stone volume showed the highest predictive value $\left(r^{2}=0.217\right)$ for successful SWL outcome on binary logistic regression analysis. Three cases that were originally classified as 'stone-free with clinically insignificant residual fragments' based on manual axis measurements actually had a larger stone volume based on CTTA estimation than the smallest fragments remaining for cases with an outcome of 'not stone-free'. This study suggests objective measurement of total stone volume could improve estimation of stone burden before and after treatment. Current definitions of stone-free status based on manual measurements of residual fragment sizes are not accurate and may underestimate remaining stone burden after treatment. Future studies reporting on the efficacy of different stone treatments should consider using objective stone volume measurements based on CT image analysis as an outcome measure of stone-free state.
\end{abstract}

Keywords Clinical insignificant residual fragments $\cdot$ Extracorporeal shockwave lithotripsy $\cdot$ Renal stone $\cdot$ Stone volume

Helen Wei Cui

helen.cui@nds.ox.ac.uk

Tze Khiang Tan

khiang94@gmail.com

Frederikke Eichner Christiansen

fec@dadlnet.dk

Palle Jörn Sloth Osther

palle.Joern.Osther@rsyd.dk

Benjamin William Turney

ben.turney@nds.ox.ac.uk

1 Oxford Stone Group, University of Oxford, Oxford, UK

2 Ninewells Hospital and Medical School, University of Dundee, Dundee DD1 9SY, UK

3 Department of Urology, Urological Research Center, Lillebaelt Hospital, University of Southern Denmark, Vejle, Denmark

\begin{tabular}{|c|c|}
\hline \multicolumn{2}{|c|}{ Abbreviations } \\
\hline CIRF & Clinically insignificant residual fragments \\
\hline CT KUB & $\begin{array}{l}\text { Computed tomography of the kidney, ureters } \\
\text { and bladder }\end{array}$ \\
\hline CTTA & Computed tomography textural analysis \\
\hline $\mathrm{HU}$ & Hounsfield unit \\
\hline IQR & Interquartile range \\
\hline PCNL & Percutaneous nephrolithotomy \\
\hline RIRS & Retrograde intrarenal surgery \\
\hline ROI & Region of interest \\
\hline WL & Extracorporeal shockwave lithotripsy \\
\hline
\end{tabular}

\section{Introduction}

Current evidence and treatment guidelines for the management of renal tract stones, including the American Urological Association (AUA), European Urology Association (EAU) and United Kingdom NICE recommendations, are 
guided by the size of the stone both at diagnosis, and of the remaining fragments after initial treatment. However, there is no agreed standardised method of defining stone size both in terms of how stone axis lengths or stone volumes should be measured, and which imaging modality should be used to visualise stone size [1-4].

Previous studies investigating the efficacy of different treatment modalities for renal tract stones have shown large heterogeneity in the methods used to define outcomes from treatment, including choice of imaging modality, method of measuring stone burden and the size definition of 'clinically insignificant residual fragments' or CIRFs [5, 6]. The recent evidence review for the NICE guidelines show the difficulties of comparing outcomes between different stone treatment modalities when the definition of 'stone-free' can vary between studies [7]. Most studies do include the presence of CIRFs as a 'stone-free' outcome after treatment, and recommendations for choosing extracorporeal shockwave lithotripsy (SWL), retrograde intrarenal surgery (RIRS) or percutaneous nephrolithotomy (PCNL) are based on these stone-free rates. However, there is no agreement on whether all CIRFs are significant and therefore only completely 'stone-free' should be included as a successful outcome of treatment, or whether some residual fragments may be more significant than others based on risk of future symptoms and need for retreatment [8].

Stone volume is now considered to be a more accurate and reliable method of measuring stone burden, which is also becoming more feasible with the increased use of computed tomography of the kidneys ureter and bladder (CT KUB) and ultra-low-dose CT KUB [9]. However, both the use of $3 \mathrm{D}$ reconstruction, and the manual calculation of stone volume using axes measurements, are not routinely performed [10]. Single axis measurement of stone burden is still the norm in clinical practice, and forms the basis of studies and guidelines for renal stone management [11, 12]. Little is known on how measuring the volume of residual stones may impact on our understanding of CIRFs.

This study compared measurement of stone burden on CT KUB images using a semi-automated software tool to estimate stone volume, versus traditional methods of estimating stone size using manual axis measurements, both before and after SWL treatment. The ability of stone volume to help predict for SWL outcome, and the significance of residual fragments based on stone axis versus volume measurement was also evaluated.

\section{Patients and methods}

Retrospective data collection of consecutive patients who had undergone SWL at a single institution between 2010 and 2014 were screened. Inclusion criteria were adult patients with renal calculi treated with SWL who had a CT KUB performed before and after SWL treatment. This is same cohort used in previous study by Christiansen et al. [13].

\section{Manual measurements of stone size}

CT KUB DICOM files were analysed using MicroDICOMviewing software. The measure tool was used to manually measure stone axis lengths. The axial slice that subjectively had the largest area was chosen, and the maximum diameter in the $x$ and $y$ perpendicular axes, and $x$ axis on coronal view were used. Manual calculation of stone volume was estimated from the following three equations for a volume of an ellipsoid: the scalene ellipsoid formula $(\pi / 6 \times a \times b$ $\times c)$, the oblate ellipsoid formula $(\pi / 6 \times a \times a \times c)$ and the prolate ellipsoid formula $(\pi / 6 \times a \times b \times b)$, where $a$ is the equatorial diameter, $b$ is the polar diameter, and $c$ is the third measurable diameter [10].

\section{Semi-automated measurements of stone size}

DICOM files were also analysed using proprietary CT texture analysis (CTTA) software (StoneChecker Software Limited, Radstock, UK) [14]. This software semi-automatically populates the region of interest (ROI) pertaining to the stone on all axial images that include the stone. This is performed by setting a Hounsfield unit (HU) threshold that would discriminate for any pixels that could represent the stone rather than surrounding tissue or urine. The pixels contained in the ROI across all image slices containing the stone are used to calculate measures of stone burden including the major horizontal axis length, major vertical axis length, cross-sectional area of the largest slice, the volume of each stone and the total number of pixels present (Fig. 1). The volume is estimated by the software by counting all the available pixels in all the ROIs drawn for the stone and multiplying this by the size of the pixel and slice thickness as obtained from the DICOM metadata.

\section{SWL treatment}

SWL treatment was performed by an experienced urologist or uroradiologist using the Medical Modulith ${ }^{\circledR}$ SLX F2 (Storz Medical AG, Switzerland) lithotriptor. During the procedure, fluoroscopy and/or ultrasonography was 


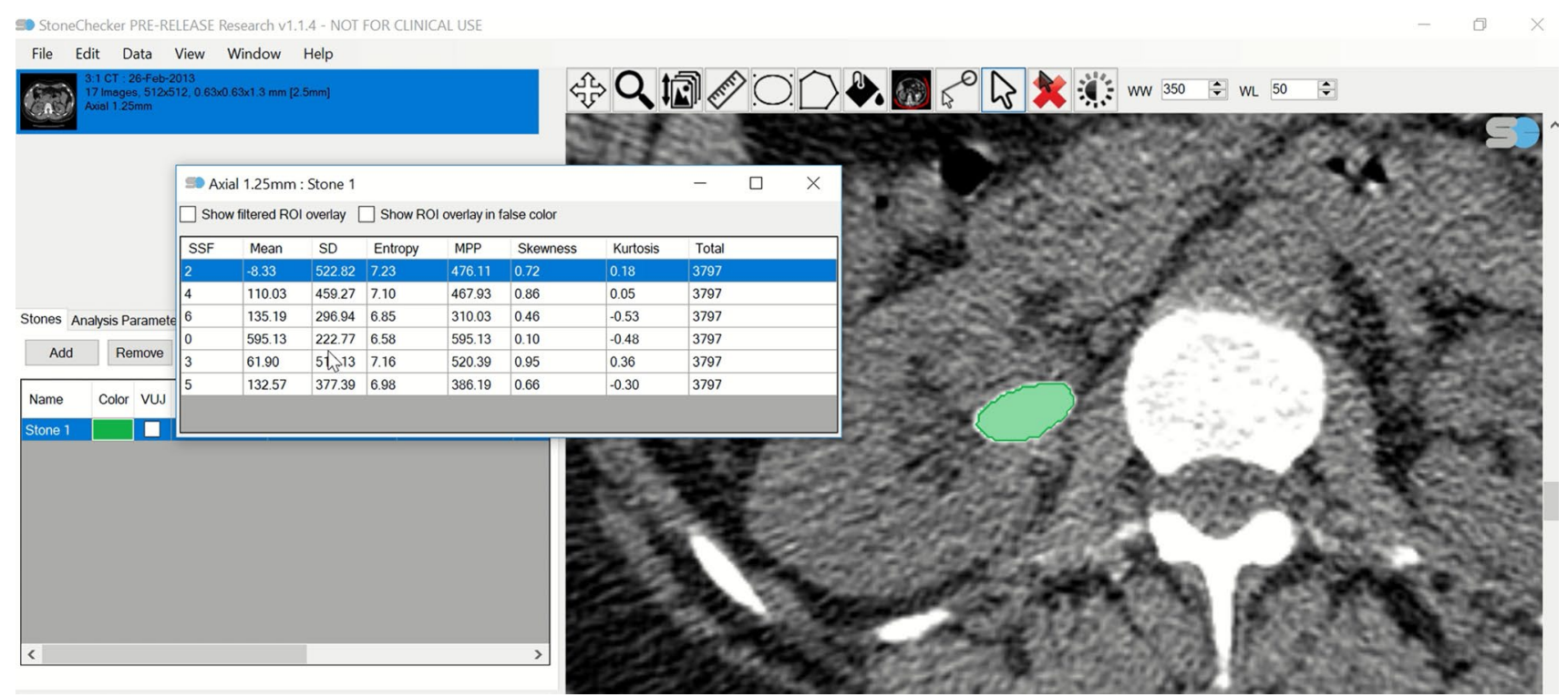

Fig. 1 Example of the generation of computed tomography textural analysis variables using the StoneChecker Software. The green area represents the semi-automated region of interest generated by the software

used for stone targeting using the 'extended focus' setting. The energy of the shock waves started at $1.0 \mathrm{~J}$ and slowly increased to the maximum energy level of $6.0 \mathrm{~J}$ depending on the stone. The number of shock waves delivered ranged from 800 to 4000 depending on the response seen on imaging at the time of treatment. Most patients received 2000-3000 shock waves at a frequency of 1.0-1.5 Hz.

\section{Outcome of SWL}

Outcome of SWL, as defined by the clinical team was based on post-treatment CT KUB imaging and measurement of any remaining fragments seen using either manual measurement or CTTA software. 'Stone-free' was defined as no stones remaining after SWL on follow-up CT KUB imaging. CIRFs were defined by the host institution as fragments $\leq 4 \mathrm{~mm}$. There was no prescribed way of measuring size of residual fragments by the host institution [10].

\section{Statistical analysis}

The strength of the relationship between the manual versus the semi-automated stone burden measurements was assessed using Pearson $r$ correlation. Binary logistic regression was performed for the degree of variance in the SWL treatment outcome explained by different measurements of stone burden. All analyses were performed using IBM SPSS Statistics for Windows, version 24.

\section{Results}

\section{Patient characteristics}

72 patients undergoing SWL were included in this study for analysis as outlined in Table 1. 69/72 (96\%) of SWL treatments were for 1 stone in the same location. The median major axis length was $6.92 \mathrm{~mm}(\mathrm{IQR}=5.7-8.9)$, and the median volume was $113 \mathrm{~mm}^{3}$ (IQR $=62-276$ ). Most of the stones were located in the lower pole (45.8\%) and renal pelvis (40.3\%). Most patients (83.3\%) required only one session of SWL. The success rate of SWL defined as 'completely stone-free' on CT KUB followup imaging was $44.4 \%$. Follow-up CT KUB to determine outcome of SWL was performed at a median of 61 days $(\mathrm{IQR}=42-76)$ after the last SWL treatment. 11/72 (15\%) of cases required additional treatment which included 10 cases requiring RIRS and one case requiring PCNL.

\section{Manual vs. semi-automated measurements of stone burden on CT KUB}

Comparison of semi-automated volume estimation using the StoneChecker software versus the manual estimations of stone volume showed that all stone size and volume variables (across the three equations used for ellipsoid volume calculation) showed strong positive correlation between these two methods (Table 2), with a Pearson correlation coefficient of $r>0.8$. More specifically, manual stone volume calculation based on the oblate and scalene 
Table 1 Demographic and clinical outcome

\begin{tabular}{|c|c|c|c|c|}
\hline & \multirow[t]{2}{*}{ Total } & \multicolumn{3}{|l|}{ Outcome } \\
\hline & & Completely stone-free & Stone-free with CIRFs & Not stone-free \\
\hline No. treated cases & 72 & $32(44)$ & $37(51)$ & $35(49)$ \\
\hline No. male (\%) & $42 / 72(58.3)$ & $20 / 32(62.5)$ & $25 / 37(67.6)$ & $18 / 35(51.4)$ \\
\hline No. left side (\%) & $34 / 72(47.2)$ & $15 / 32(46.9)$ & $19 / 37(51.4)$ & $16 / 35(45.7)$ \\
\hline \multicolumn{5}{|l|}{ No. stone location (\%) } \\
\hline Upper pole & $2 / 72(2.8)$ & $2 / 32(6.3)$ & $2 / 37(5.4)$ & \\
\hline Midpole & $7 / 72(9.7)$ & $4 / 32(12.5)$ & 4/37 (10.8) & $5 / 34(14.7)$ \\
\hline Lower pole & $33 / 72(45.8)$ & $18 / 32(56.3)$ & $21 / 37(56.8)$ & $15 / 35(42.9)$ \\
\hline Renal pelvis & $29 / 72(40.3)$ & $8 / 32(25.0)$ & $10 / 37(27.0)$ & $14 / 35(40.0)$ \\
\hline Missing & $1 / 72(1.4)$ & & & $1 / 35(2.9)$ \\
\hline \multicolumn{5}{|l|}{ Measures of stone burden } \\
\hline \multicolumn{5}{|l|}{ No. of stones } \\
\hline 1 & $69 / 72(96)$ & $32 / 32(100)$ & $36 / 37(97.3)$ & $33 / 35(94.3)$ \\
\hline 2 & $3 / 72(4)$ & & $1 / 37(2.7)$ & $2 / 35(5.7)$ \\
\hline \multicolumn{5}{|l|}{ Stone axis length } \\
\hline Major axis length; $\mathrm{mm}^{\mathrm{a}}$ & $6.9(5.7-8.9[3.4-20.1])$ & $6.2(5.2-8.1[3.4-11.8])$ & $6.3(5.2-8.1[3.4-13.8])$ & $7.7(6.0-10.8[3.9-20.1])$ \\
\hline Vertical axis length; $\mathrm{mm}^{\mathrm{a}}$ & $7.5(5.7-10.5[2.6-21.0])$ & $6.7(5.3-8.1[3.7-12.7])$ & $6.8(5.0-8.6[3.7-12.7])$ & $9.0(6.0-11.4[2.6-21.0])$ \\
\hline Stone volume $(\mathrm{s}) ; \mathrm{mm}^{3 \mathrm{a}}$ & $113(62-276$ [21-1820]) & $74(56-141[21-350])$ & $80(58-168$ [21-518]) & $199(77-396$ [8-1820]) \\
\hline Total no. of pixels ${ }^{\mathrm{a}}$ & $220(139-485[30-3224])$ & $190(131-391$ [65-966]) & $200(142-446[65-1615])$ & $238(133-511[30-3224])$ \\
\hline \multicolumn{5}{|l|}{ No. SWL sessions } \\
\hline 1 & $60 / 72(83.3)$ & $30 / 32(93.8)$ & 35/37 (94.6) & $27 / 35(77.1)$ \\
\hline 2 & $10 / 72(13.9)$ & $1 / 32(3.1)$ & $1 / 37(2.7)$ & $7 / 35(20.0)$ \\
\hline 3 & $1 / 72(1.4)$ & & & \\
\hline$\geq 4$ & $1 / 72(1.4)$ & $1 / 32(3.1)$ & 1/37 (2.7) & $1 / 35(2.9)$ \\
\hline
\end{tabular}

CIRFs, clinically insignificant residual fragments; CT KUB, computed tomography kidneys ureter bladder; SWL, shockwave lithotripsy

${ }^{a}$ Values were measured using the StoneChecker software. 'Stone-free with CIRFs' are patients who are completely stone-free or patients who had CIRFs after treatment. 'Completely stone-free' is a subset of 'Stone-free with CIRFs' for patients who are completely stone-free only. 'Not stone-free' are patients who have residual fragments after treatment which are larger than CIRFs. 'Total' includes the sum of patients who are 'Stone-free with CIRFs' and 'Not stone-free'

Table 2 Correlation between a manual versus semi-automated method of measuring stone size

\begin{tabular}{lccc}
\hline Variables & Manual method & Semi-automated method & $\begin{array}{l}\text { Pearson } r \\
\text { correlation }\end{array}$ \\
\hline $\begin{array}{c}\text { Major horizontal } \\
\text { axis length (mm) }\end{array}$ & $6.6(5.4-9.0[3.0-20.8])$ & $6.9(5.7-8.9[3.4-20.1])$ & 0.97 \\
$\begin{array}{l}\text { Major vertical axis } \\
\text { length }(\mathrm{mm})^{\mathrm{a}}\end{array}$ & $7.2(4.7-9.3[2.6-16.3])$ & $7.5(5.7-10.5[2.6-21.0])$ & 0.88 \\
$\begin{array}{l}\text { Manual volume estimation using different equations for the volume of an ellipsoid } \\
\text { Oblate }\end{array}$ & $161(71-379[13-3347])$ & \\
Scalene & $100(40-247[10-1420])$ & & 0.94 \\
Prolate & $65(24-181[1-1172])$ & 0.94 \\
\hline
\end{tabular}

Values are median (IQR [range])

${ }^{a}$ Values were measured from the largest cross-sectional slice of the stone manually. The manual method of calculating stone volume used the following formulas for an ellipsoid: oblate ellipsoid $(\pi / 6 \times a \times a \times c)$, scalene ellipsoid $(\pi / 6 \times a \times b \times c)$ and prolate ellipsoid $(\pi / 6 \times a \times b \times b)$ 
formulas for an ellipsoid showed correlation with software estimated volume of $r>0.9$.

\section{Classification of CIRFs usual manual vs. semi-automated size measurements}

Outcome of SWL was classified in one of three categories: 'completely stone-free', 'stone-free with CIRFs' and 'not stone-free' based on the original classification using manual stone axis measurements of follow-up CT KUB images. For cases with an outcome of 'stone-free with CIRFs' and 'not stone-free', the total volume of any remaining fragments was estimated using the StoneChecker software. Based on volume estimation using StoneChecker, there was an overlap of stone volumes between the 'not stone-free' and 'stonefree with CIRFs' groups (Table 3). There were three stones that were originally classified as 'stone-free with CIRFs' which were subsequently found to have a total stone volume larger than the volume of the smallest fragments $\left(8.34 \mathrm{~mm}^{3}\right)$ found in the 'not stone-free' group. This showed that the original classification of treatment outcome as "stone-free with CIRFs' may have underestimated the total remaining stone burden of some cases. Conversely, measurement of major axis length showed that three cases with an original outcome of 'not stone-free' had a major axis diameter of less than $4 \mathrm{~mm}$ which would therefore have qualified to have an outcome of 'stone-free with CIRFs'.

\section{Volume vs axis length for predicting stone-free outcome}

Traditionally, the major horizontal axis length serves as the predictive parameter for the outcome of SWL. As can be expected, the cases in this study which showed an outcome of 'completely stone-free', overall, had a smaller major horizontal axis length than those cases with a treatment outcome of 'not stone-free'. Analysis of the stone size variables for the outcome of SWL using binary logistic regression demonstrated a significant contribution from StoneChecker measured variables of stone volume, major axis length, vertical

Table 3 Major horizontal length and volume of the five smallest stone fragments remaining in the 'not stone-free' (a) and 'stone-free with CIRFs' groups (b) measured using the StoneChecker software.

Results in bold in section (b) show that 3 cases classified as "stonefree with CIRFs' had a larger volume than the smallest volume in the 'not stone-free group'

(a) Post-SWL remaining stone size and volume in the 'not stone-free' group

\begin{tabular}{lr}
\hline Major horizontal length $(\mathrm{mm})$ & ${\text { Volume }\left(\mathrm{mm}^{3}\right)}$ \\
\hline 2.387 & 8.34 \\
2.535 & 10.61 \\
3.516 & 10.78 \\
4.238 & 16.18 \\
4.386 & 19.11 \\
\hline
\end{tabular}

(b) Post-SWL remaining stone size and volume in the 'stone-free with CIRFs' group

\begin{tabular}{lr}
\hline Major horizontal length $(\mathrm{mm})$ & ${\text { Volume }\left(\mathrm{mm}^{3}\right)}^{3}$ \\
\hline 1.642 & 2.38 \\
2.304 & 7.05 \\
3.516 & $\mathbf{1 0 . 3 0}$ \\
3.672 & $\mathbf{1 1 . 7 3}$ \\
3.854 & $\mathbf{2 6 . 0 7}$ \\
\hline
\end{tabular}

CIRF, clinical insignificant residual fragment; SWL, extracorporeal shock wave lithotripsy

Table 4 Comparison of the prediction ability for an outcome of 'stone-free' after extracorporeal shock wave lithotripsy using the volume and stone axis measurements generated by StoneChecker

\begin{tabular}{llllll}
\hline Variables & Sig & Exp $(B)$ & \multicolumn{2}{l}{$95 \%$ CI for Exp $(B)$} & $\begin{array}{l}\text { Nagelkerke } \\
R \text { Square }\end{array}$ \\
\cline { 5 - 6 } & & & Lower & Upper & \\
\hline Major axis length $(\mathrm{mm})$ & 0.036 & 0.806 & 0.66 & 0.986 & 0.099 \\
Vertical axis length $(\mathrm{mm})$ & 0.034 & 0.828 & 0.696 & 0.986 & 0.100 \\
Area of largest slice/ROI $\left(\mathrm{mm}^{2}\right)$ & 0.014 & 0.964 & 0.936 & 0.993 & 0.156 \\
Stone volume $\left(\mathrm{mm}^{3}\right)$ & 0.008 & 0.994 & 0.99 & 0.999 & 0.217 \\
\hline
\end{tabular}

The higher value of Nagelkerke $R$ Square indicates better prediction of stone-free rate outcome $\mathrm{CI}$, confidence interval; ROI, region of interest 
axis length, slice area; as well as significant contributions from manually measured variables of minor axis length and stone volume (Table 4). However, the CTTA measurements of stone volume had the highest statistical predictive value as compared to the rest of the parameters (Nagelkerke $R$ square $=0.217$ ). This indicates that stone volume variable can explain $21.7 \%$ of the variance in the outcome of stonefree or not. In comparison, major axis length as measured using StoneChecker showed the ability to explain $9.9 \%$ of the variance in the outcome of stone-free or not.

\section{Discussion}

In this study, measurement of stone volume using an objective, semi-automated CTTA software programme provides more information on stone burden before and after SWL treatment. Before treatment, stone volume has more predictive ability for successful SWL outcome than axis measurements. After SWL, stone burden can be underestimated if based on axis measurements of fragments alone. The total volume of fragments may be more helpful to determine whether any residual fragments are significant and likely to need retreatment.

As CT becomes the standard imaging modality to diagnose renal tract stones, the utility of measuring stone volume using CT software is increasingly recognised as important for planning treatment and predicting treatment outcome. Previous studies have used several different methods for estimating stone volume using CT images including, 3D reconstruction of the stone to measure axis lengths and then applying an ellipsoid formula $[9,15]$; using third party CT software to estimate volume $[10,16]$; and using a HU thresholding and voxel counting technique [17]. Using stone volume instead of axial measurements may be a better predictor of treatment outcome, as small differences in manual axis measurements may lead to much larger volume changes $[9,16]$. This may be more applicable with increasing size of stone, as found by Finch et al. [10]. As maximum stone diameter increases, stone volume estimation using ellipsoid volume equations become less accurate [10]. These results reflect our findings as shown in Table 2 of the ellipsoid equation volume estimation versus the semi-automated volume estimation. The manual volume estimates in our study vary significantly depending on which equation is used, although the relationship between manual and automated methods is strong across all equations [10]. The technique in this study also employs voxel counting as well as automated selection of the region of interest based on HU thresholding. This has advantages over other techniques which require manual drawing of the region of interest or manual measurement of axis lengths which may be inaccurate and are less reproducible [16].
Stone size has been shown in previous studies to be a strong predictor for SWL outcome amongst other stone and patient related factors [18-23]. In almost all studies, a measurement of stone axis diameter is used. Our study supports previous evidence that stone volume is a stronger predictor of SWL outcome than axis length [9]. On binary logistic regression, using StoneChecker measurements, stone volume explains slightly more of the variation in the SWL treatment outcome of stone-free or not than the major axis length (Nagelkerke $R$ squared of 0.099 for major axis length compared to 0.217 for stone volume). Although estimated volume using the CTTA software did correlate highly with manually estimated volume $(r>0.9)$, the use of software volume calculation is likely to be less arduous than manual measurement of 3 stone axes lengths and, our analysis suggests the CTTA estimated volume is slightly more predictive for outcome than manually estimated volume. This technique may be especially useful for larger stones with irregular outlines, or where there is more than one stone, as the software can sum the volumes of all stones to be treated.

The utility of stone volume measurement to plan treatment is already recognised as important [24]. However, less has been studied on the volume measurement of residual fragments after treatment. In this study, three stones that were originally classified as 'stone-free with CIRFs' actually had a total stone volume larger than the volume of the smallest residual fragments in the 'not stone-free group'. This shows that classifying stone outcome as 'stone-free with CIRFs' can lead to misclassification of some stones that are actually larger than those classified as 'not stone-free'. Accurate stone volume measurement of residual fragments may have more value in defining outcome from stone treatment $[17,25]$. This study showed an overlap of stone volumes between cases classified as 'stone-free with CIRFs' and 'not stone-free', i.e. had residual fragments after treatment that were $>4 \mathrm{~mm}$ and therefore not CIRFs. For example, one of the cases of 'not stone-free' had a residual fragment with a major horizontal length of $4.238 \mathrm{~mm}$ and stone volume of $16.18 \mathrm{~mm}^{3}$, whereas a case of 'stone-free with CIRFs' had a major horizontal length of $3.854 \mathrm{~mm}$ and a larger stone volume of $26.07 \mathrm{~mm}^{3}$.

Defining outcome of stone treatment based on 'completely stone-free' or 'not stone-free has been advocated by some, to reduce confusion over the use of the term CIRFs [26]. However, studies of the natural history of CIRFs after SWL and RIRS have shown that that the majority of CIRFs (approx. 56-78\%) do spontaneously pass or requires no further treatment [8, 26-29]. This still leaves a significant proportion (approx. 29-50\%) which does require retreatment. A recent study followed 232 subjects after RIRS who had residual fragments of any size for 12 months with the availability of follow-up imaging. This found that $29 \%$ of subjects required intervention 
during the follow-up period and likelihood of reintervention was predictable based on fragment size [27]. Given that most cases do not require reintervention for CIRFs, removing this term entirely may not be helpful for clinical decision making on which patients may require a secondary intervention after initial SWL. It is likely there is close relationship between size of residual fragments and need for reintervention and therefore accurate measurement of residual fragment size is important. This can help to both compare treatment outcomes in studies of stone interventions and inform further research on predicting which residual fragments are likely to require further treatment.

The authors acknowledged that this study possesses limitations. Firstly, this study is retrospective in nature and patients could only be included who had a CT both before and after SWL treatment. This may have biased to include a population of patients who had a lower success rate from SWL as most straightforward cases of SWL do not usually require $\mathrm{CT}$ after treatment. The timing of the post-treatment CT was therefore not standardised, and the presence and size of residual stone fragments would be influenced by time passed after SWL treatment. However, this does not change the method by which remaining stone fragments after SWL were measured. Secondly, there was a lack of standardisation of the original method of measuring CIRFs by the clinical team which leads to exaggerated inaccuracies in stone burden estimation after treatment. Thirdly, the sample size in this study is small due to the data available from this single centre. It is difficult to draw conclusions on how stone size is estimated in other centres to understand the applicability of these results. However, of the stone studies in the literature, stone size measurement is often not reported in the methodology as a standardised method.

\section{Conclusion}

This study shows the potential benefit of objective measurements of stone burden using a semi-automated approach both before, and after, SWL treatment. This method of measuring stone volume is less time consuming than manual methods and, offers a more standardised approach to comparing the efficacy of different stone treatments as part of clinical trials.

Author contributions FEC and PJSO collected the original data and contributed to the design of the study and review of the manuscript. TKT performed analysis of the data and writing of the manuscript. HWC designed the study, performed analysis of the study and writing of the manuscript. BWT contributed to the design of the study and review of the manuscript.
Funding None.

Code availability Not applicable.

\section{Compliance with ethical standards}

Conflict of interest All the authors declare no conflicts of interest.

Ethics approval Approved study by the Department of Urology, Lillebaelt Hospital, University of Southern Denmark, Denmark.

Availability of data and material Results of the data collection of stone measurements and CTTA results are available.

Open Access This article is licensed under a Creative Commons Attribution 4.0 International License, which permits use, sharing, adaptation, distribution and reproduction in any medium or format, as long as you give appropriate credit to the original author(s) and the source, provide a link to the Creative Commons licence, and indicate if changes were made. The images or other third party material in this article are included in the article's Creative Commons licence, unless indicated otherwise in a credit line to the material. If material is not included in the article's Creative Commons licence and your intended use is not permitted by statutory regulation or exceeds the permitted use, you will need to obtain permission directly from the copyright holder. To view a copy of this licence, visit http://creativecommons.org/licenses/by/4.0/.

\section{References}

1. Türk C, Skolarikos A, Neisius A et al (2019) EAU guidelines on urolithiasis. European Association of Urology. https://uroweb.org/ wp-content/uploads/EAU-Guidelines-on-Urolithiasis-2019.pdf. Accessed 11 Feb 2020

2. Assimos D, Krambeck A, Miller NL, Monga M, Murad MH, Nelson CP et al (2016) Surgical management of stones: American Urological Association/Endourological Society Guideline, Part I. J Urol 196:1153-1160. https://doi.org/10.1016/j.juro.2016.05.090

3. Assimos D, Krambeck A, Miller NL, Monga M, Murad MH, Nelson CP et al (2016) Surgical management of stones: American Urological Association/Endourological Society Guideline, Part II. J Urol 196:1161-1169. https://doi.org/10.1016/j.juro.2016.05.091

4. Guideline NICE (2019) Renal and ureteric stones: assessment and management: NICE (2019) renal and ureteric stones: assessment and management. BJU Int 123(2):220-232. https://doi. org/10.1111/bju.14654

5. Hyams ES, Bruhn A, Lipkin M, Shah O (2010) Heterogeneity in the reporting of disease characteristics and treatment outcomes in studies evaluating treatments for nephrolithiasis. J Endourol 24(9):1411-1414. https://doi.org/10.1089/end.2009.0645

6. Cui HW, Turney BW (2019) Variability and quality of outcome reporting in clinical trials of interventions for renal calculi. J Clin Urol 12(15):11

7. NICE guideline [NG118] (2020) Renal and ureteric stones: assessment and management. https://www.nice.org.uk/guidance/ng118. Accessed 11 Feb 2020

8. Streem SB, Yost A, Mascha E (1996) Clinical implications of clinically insignificant store fragments after extracorporeal shock wave lithotripsy. J Urol 155(4):1186-1190. https://doi. org/10.1016/S0022-5347(01)66208-6

9. Bandi G, Meiners RJ, Pickhardt PJ, Nakada SY (2009) Stone measurement by volumetric three-dimensional computed tomography for predicting the outcome after extracorporeal shock wave 
lithotripsy. BJU Int 103(4):524-528. https://doi.org/10.1111/ j.1464-410X.2008.08069.x

10. Finch W, Johnston R, Shaida N, Winterbottom A, Wiseman O (2014) Measuring stone volume-three-dimensional software reconstruction or an ellipsoid algebra formula? BJU Int 113:610 614. https://doi.org/10.1111/bju.12456

11. McClinton S, Cameron S, Starr K, Thomas R, MacLennan G, McDonald A, Lam T, N'Dow J, Kilonzo M, Pickard R, Anson K (2018) TISU: extracorporeal shockwave lithotripsy, as first treatment option, compared with direct progression to ureteroscopic treatment, for ureteric stones: study protocol for a randomised controlled trial. Trials 19(1):1. https://doi.org/10.1186/s1306 3-018-2652-1

12. Sener NC, Bas O, Sener E, Zengin K, Ozturk U, Altunkol A, Evliyaoglu Y (2015) Asymptomatic lower pole small renal stones: shock wave lithotripsy, flexible ureteroscopy, or observation? A prospective randomized trial. Urology 85(1):33-37. https://doi. org/10.1016/j.urology.2014.08.023

13. Christiansen FE, Andreassen KH, Osther SS, Osther PJ (2016) Internal structure of kidney calculi as a predictor for shockwave lithotripsy success. J Endourol 30(3):324. https://doi.org/10.1089/ end.2015.0616

14. Cui HW, Devlies W, Ravenscroft S, Heers H, Freidin AJ, Cleveland RO, Ganeshan B, Turney BW (2017) CT texture analysis of ex vivo renal stones predicts ease of fragmentation with shockwave lithotripsy. J Endourol 31(7):694-700. https://doi. org/10.1089/end.2017.0084

15. Ito H, Kawahara T, Terao H, Ogawa T, Yao M, Kubota Y, Matsuzaki J (2012) The most reliable preoperative assessment of renal stone burden as a predictor of stone-free status after flexible ureteroscopy with holmium laser lithotripsy: a single-center experience. Urology 80(3):524-528. https://doi.org/10.1016/j.urolo gy.2012.04.001

16. Patel SR, Stanton P, Zelinski N, Borman EJ, Pozniak MA, Nakada SY, Pickhardt PJ (2011) Automated renal stone volume measurement by noncontrast computerized tomography is more reproducible than manual linear size measurement. J Urol 186(6):22752279. https://doi.org/10.1016/j.juro.2011.07.091

17. Yoshida S, Hayashi T, Morozumi M, Osada H, Honda N, Yamada $\mathrm{T}$ (2007) Three-dimensional assessment of urinary stone on noncontrast helical computed tomography as the predictor of stonestreet formation after extracorporeal shock wave lithotripsy for stones smaller than $20 \mathrm{~mm}$. Int J Urol 14(7):665-667. https://doi. org/10.1111/j.1442-2042.2007.01767.x

18. Cui HW, Silva MD, Mills AW, North BV, Turney BW (2019) Predicting shockwave lithotripsy outcome for urolithiasis using clinical and stone computed tomography texture analysis variables. Sci Rep 9(1):14674. https://doi.org/10.1038/s41598-019-51026-x

19. Cohen TD, Preminger GM (1997) Management of calyceal calculi. Urol Clin N Am 24(1):81-96. https://doi.org/10.1016/s0094 $-0143(05) 70356-6$
20. Abdel-Khalek M, Sheir KZ, Mokhtar AA, Eraky I, Kenawy M, Bazeed M (2004) Prediction of success rate after extracorporeal shock-wave lithotripsy of renal stones. A multivariate analysis model. Scand J Urol Nephrol 38(2):161-167. https://doi. org/10.1080/00365590310022626

21. Lalak NJ, Moussa SA, Smith G, Tolley DA (2002) The Dornier Compact Delta lithotripter: the first 500 renal calculi. J Endourol 16(1):3-7. https://doi.org/10.1089/089277902753483637

22. Sorensen CM, Chandhoke PS (2002) Is lower pole calyceal anatomy predictive of extracorporeal shock wave lithotripsy success for primary lower pole kidney stones? J Urol 168(6):2377-2382. https://doi.org/10.1097/01.ju.0000036354.52323.c1

23. Guler Y, Erbin A, Kafkasli A, Ozmerdiven G (2020) Factors affecting success in the treatment of proximal ureteral stones larger than $1 \mathrm{~cm}$ with extracorporeal shockwave lithotripsy in adult patients. Urolithiasis. https://doi.org/10.1007/s00240-02001186-7

24. Chaussy CG, Tiselius HG (2018) How can and should we optimize extracorporeal shockwave lithotripsy? Urolithiasis 46(1):317. https://doi.org/10.1007/s00240-017-1020-z

25. Olcott EW, Sommer FG, Napel S (1997) Accuracy of detection and measurement of renal calculi: in vitro comparison of three-dimensional spiral CT, radiography, and nephrotomography. Radiology 204(1):19-25. https://doi.org/10.1148/radio logy.204.1.9205217

26. Khaitan A, Gupta NP, Hemal AK, Dogra PN, Seth A, Aron M (2002) Post-ESWL, clinically insignificant residual stones: reality or myth? Urology 59(1):20-24. https://doi.org/10.1016/s0090 $-4295(01) 01494-7$

27. Chew BH, Brotherhood HL, Sur RL, Wang AQ, Knudsen BE, Yong C, Marien T, Miller NL, Krambeck AE, Charchenko C, Humphreys MR (2016) Natural history, complications and reintervention rates of asymptomatic residual stone fragments after ureteroscopy: a report from the EDGE research consortium. J Urol 195(4 Pt 1):982-986. https://doi.org/10.1016/j.juro.2015.11.009

28. Osman MM, Alfano Y, Kamp S, Haecker A, Alken P, Michel MS, Knoll T (2005) 5-year-follow-up of patients with clinically insignificant residual fragments after extracorporeal shockwave lithotripsy. Eur Urol 47(6):860-864. https://doi.org/10.1016/j. eururo.2005.01.005

29. Pullar B, Lunter C, Collie J, Shah S, Shah N, Hayek S, Wiseman OJ (2017) Do renal stones that fail lithotripsy require treatment? Urolithiasis 45(6):597-601. https://doi.org/10.1007/s0024 0-017-0973-2

Publisher's Note Springer Nature remains neutral with regard to jurisdictional claims in published maps and institutional affiliations. 